\section{Super-PTEN mice}

Loss of function of the tumor suppressor PTEN frequently occurs in cancer. Whereas overexpression of PTEN in Drosophila causes embryonic lethality, the effect of overexpression in the mouse was unknown. Pier Paolo Pandolfi and colleagues report that increased PTEN levels unexpectedly result in viable mice that display a tumor-resistant, anti-Warburg metabolic state (Cell 149, 49-62, 2012). Transgenic Super-PTEN mice carrying the entire Pten genomic locus were constructed and found to express Pten at 1.1- to 3.5-fold higher levels than normal mice. These mice had decreased body weight and size due to decreases in cell size and number. Using chemical carcinogen-induced models of cancer, the authors found that Super-PTEN mice showed a significantly longer latency of tumor formation. Super-PTEN mice also showed an increase in energy expenditure and reduced body fat compared to wild-type mice. Cells overexpressing PTEN had decreased phosphatidylinositol 3-kinase (PI3K) activity and consumed less glucose than wild-type cells. These results indicate that PTEN overexpression leads to a reduction in PI3K-Akt signaling. Although cells from Super-PTEN mice used less glucose, they showed higher mitochondrial oxidative phosphorylation, which is consistent with an anti-Warburg state. The authors suggest that this metabolic state contributes to cancer resistance in these mice, and elevation of PTEN levels may be a potential therapeutic approach in cancer.

\section{Familial diarrhea syndrome}

Torunn Fiskerstrand and colleagues report an activating mutation in GUCY2C as the cause of an autosomal dominant diarrhea syndrome in a large Norwegian family (N. Engl. J. Med., published online 21 March 2012; doi:10.1056/NEJMoa1110132). The authors mapped the disease locus to a $2.9-\mathrm{Mb}$ region on chromosome 12 by using linkage analysis. They then sequenced the most promising candidate gene in the region, GUCY2C, which encodes a transmembrane guanylate cyclase that functions as an intestinal receptor for bacterial endotoxins, and identified a missense variant (encoding p.Ser840Ile) that co-segregated with the disease in the family. The altered residue is located in the protein's catalytic domain and leads to higher guanylate cyclase activity following ligand stimulation, resulting in increased cyclic GMP production. The authors propose that the elevated cyclic GMP levels stimulate activity of the CFTR chloride channel, resulting in increased water efflux into the intestinal lumen. The authors note that the disease symptoms in this family overlap with those seen in other diseases, including Crohn's disease and irritable bowel syndrome, suggesting that deregulation of this guanylate cyclase pathway could contribute to more common forms of inflammatory bowel disease.

KV

\section{Straightforward recessive BBS}

Bardet-Biedl syndrome is a heterogeneous, multisystem disorder of ciliary function with variable expressivity within families. One model for incomplete penetrance invokes recessive inheritance with a heterozygous modifier at a second locus. Leen Abu-Safieh and colleagues now provide direct evidence against this model by using the most direct experimental approach (Eur. J. Hum. Genet. 20, 420-427, 2012). They sequenced

Written by Myles Axton, Orli Bahcall, Emily Niemitz, Pamela Feliciano, \& Kyle Vogan the entire coding and flanking intronic regions of BBS1 to BBS14 in all affected individuals and their unaffected relatives from 29 families with Bardet-Biedl syndrome, finding only a homozygous mutation in a single gene in each affected individual and no evidence for third alleles. Homozygous mutations were not found in unaffected individuals. The study families were, with just one exception, consanguineous, but the allelic and locus heterogeneity found argues that the results are likely to apply to other populations. The authors note that their $100 \%$ rate of mutation detection is much higher than that of foregoing studies. Cryptic splicing mutations can be missed by DNA sequencing approaches, and their presence in compound heterozygotes can lead to the erroneous conclusion of triallelic inheritance.

$M A$

\section{Interpretation of regulatory polymorphism}

Approaches to functionally interpret disease-associated common noncoding genetic variants are needed. Now, John Stamatoyannopoulos and colleagues report an analysis to determine how genetic variation affects the activity of the CTCF DNA-binding factor (PLoS Genet. 8, e1002599, 2012). The authors mapped CTCF-binding sites across the genome by chromatin immunoprecipitation followed by sequencing (ChIP-seq) in transformed B-lymphoblastoid cells from 12 members of a 3-generation pedigree, and then performed targeted resequencing of these sites to catalog genetic variation. Of 35,709 CTCF-binding sites, $21 \%$ contained one or more SNP, and integration of the CTCF-binding and genetic variation data sets yielded 325 sites at which a SNP genotype was associated with a quantitative measure of CTCF occupancy. The majority of SNPs that affected CTCF occupancy were located in regions of protein-DNA contact, but these SNPs represent only a small minority of all SNPs located in regions of CTCF binding, indicating that there is context-dependent buffering of the ability of genetic variation to alter CTCF occupancy. The authors further found that buffering is partly determined by the strength of the CTCF-binding site and by local sequence context. These findings indicate that local context effects will complicate efforts to predict functional effects of noncoding SNPs.

EN

\section{Chimpanzee genetic map}

Gil McVean and colleagues report a fine-scale chimpanzee genetic map constructed using a whole-genome population sequencing approach (Science, published online 15 March 2012; doi: $10.1126 /$ science.1216872). They sequenced the genomes of 10 unrelated Western Chimpanzees (Pan troglodytes verus) with an average 9.1-fold coverage. They identified 5.3 million autosomal SNPs and inferred variants and haplotypes with high accuracy, which was further improved using a new regional filtering approach. They used this genetic variation data set to estimate the chimpanzee genetic map. At a broad scale, they found that the recombination rates across entire chromosomes were similar in chimpanzees and humans. The notable exceptions were regions with chromosomal rearrangement and chromosome 2, at a historic telomeric fusion event. Fine-scale recombination rates showed less conservation, with no recombination hotspots shared in humans and chimpanzees. However, some general patterns remained similar, including an increased average fine-scale recombination rate around genes and $\mathrm{CpG}$ islands. The authors also sequenced 48 PRDM9 alleles in Western Chimpanzees and found high levels of genetic variation. They did not identify any DNA motifs, repeat elements or predicted PRDM9-binding sites consistently associated with recombination hotspots in chimpanzees. 